\title{
Particular gene upregulation and p53 heterogeneous expression in TP53-mutated maxillary carcinoma
}

\author{
ITSUHIRO KUDO $^{1,2}$, MARIKO ESUMI ${ }^{1}$, YOSHIAKI KUSUMI ${ }^{1}$, TOHRU FURUSAKA ${ }^{2}$ and TAKESHI OSHIMA $^{2}$ \\ Departments of ${ }^{1}$ Pathology and ${ }^{2}$ Otorhinolaryngology-Head and Neck Surgery, \\ Nihon University School of Medicine, Tokyo 173-8610, Japan
}

Received September 14, 2016; Accepted August 3, 2017

DOI: $10.3892 / \mathrm{ol} .2017 .6751$

\begin{abstract}
It has been demonstrated that tumor protein p53 (TP53) mutation in maxillary squamous cell carcinoma, is more treatment-resistant compared with the carcinoma without TP53 mutation. However, the association between TP53 mutation and treatment resistance remains unclear. As a first step in understanding the biological differences between tumors with and without TP53 mutation, a comprehensive gene expression analysis of maxillary squamous cell carcinoma with or without TP53 mutation was performed. A total of 42 genes were identified to be differentially expressed by $>4$-fold. Quantification of their mRNA using quantitative polymerase chain reaction indicated 18 genes with high expression and three genes with low expression in TP53 mutated tumors vs. TP53 wild-type tumors. The 18 genes included eight cell adhesion (DSC3, GRHL1, EPPK1, PROM2, ANXA8, DSP, JUP, and KRT6B) and four cell growth inhibition (SFN, CLCA2, SAMD9 and TP63) genes. Among these genes, DSC3, SFN, and CSTA, whose expression was markedly increased, also demonstrated high protein expression in immunohistochemical staining of TP53 mutated tumors. The TP53 mutated tumors demonstrated high nuclear staining of the TP53 protein only in tumor cells at the tumor margins adjacent to the stroma, whereas the tumor interior was negative for TP53. However, all tumor cells of TP53 wild-type tumors exhibited positive nuclear staining for the TP53 protein. The combined findings suggest that TP53 mutated tumors possess a phenotype opposite to that
\end{abstract}

Correspondence to: Dr Mariko Esumi, Department of Pathology, Nihon University School of Medicine, 30-1 Ohyaguchikami-cho, Itabashi-ku, Tokyo 173-8610, Japan

E-mail: esumi.mariko@nihon-u.ac.jp

Abbreviations: PCR, polymerase chain reaction; CDDP, cisplatin; TP53, tumor protein p53; HNSCC, head and neck squamous cell carcinoma; TP63, tumor protein p63; IHC, immunohistochemistry; CSTA, cystain A; SFN, stratifin; DSC3, desmocollin 3; DSP, desmoplakin; JUP, junction plakoglobin; ECM, extracellular matrix

Key words: gene expression, maxillary cancer, microarray, mutation, TP53 associated with cancer progression and malignant transformation, and exhibit tumor cell heterogeneity between the tumor interior and margins.

\section{Introduction}

Maxillary cancer is commonly treated with cisplatin (CDDP) chemotherapy combined with radiotherapy. Although the tumor regresses during treatment, in about half of the cases the tumor histopathologically remains, and ultimately the CDDP-resistant tumor is surgically resected. CDDP plays a central role in treatment of maxillary cancer; however, the existence of a CDDP-resistance mechanism has now been recognized (1). To examine whether known chemotherapy-resistant genes are involved in CDDP resistance of maxillary carcinoma, we previously analyzed gene expression in maxillary squamous cell carcinoma biopsies prior to treatment. The results showed that expression of a group of genes (multidrug resistance protein 1; multidrug resistance associated protein $1 ; \mathrm{Cu}^{++}$transporting, beta polypeptide; xeroderma pigmentosum; complementation group A; excision repair cross-complementing rodent repair deficiency, complementation group 1; B-cell CLL/lymphoma 2) associated with treatment resistance was decreased in these tumors, and that only tumor protein p53 (TP53) mutation was linked to treatment resistance (2). TP53 mutations are associated with treatment resistance not only in head and neck cancers, but also in breast cancer, lung cancer, hepatic cancer, and chronic lymphocytic leukemia (3-8). On the other hand, it has been reported that there is no such association in small cell lung cancer or epithelial ovarian cancer $(9,10)$. Thus, the relationship between TP53 mutation and treatment resistance is not necessarily clear.

Recently, whole exome sequencing has shown major driver genes in head and neck squamous cell carcinoma (HNSCC). In addition to the previously identified TP53, cyclin dependent kinase inhibitor 2A, Phosphatidylinositol-4,5-bisphosphate 3-kinase catalytic subunit alpha, and histidyl-tRNA synthetase genes, mutations in major genes that regulate squamous differentiation, including notch1, interferon regulatory factor 6, and tumor protein p63 (TP63), have been newly identified as drivers $(11,12)$. In particular, TP53 mutations occur at a high frequency in HNSCC, but many non-TP53 mutated tumors are human papillomavirus-positive (13). Both types of tumors may 
involve a common mechanism mediated by TP53 dysfunction, but the biological differences between these cancers are unclear. As a first step in understanding the biological differences observed between tumors with and without TP53 mutation, this study aimed to clarify differences at the gene expression level between maxillary cancers with and without TP53 mutation.

\section{Materials and methods}

Samples. Specimens were used from 14 patients with maxillary cancer (Table I). Tumor staging and differentiation was in accordance with the Union for International Cancer Control TNM classification (14). Maxillary cancer biopsy specimens before treatment were used in the study. This study was approved by the Ethics Committee at Nihon University School of Medicine and conforms to the Declaration of Helsinki (2013). Informed consent was obtained from all patients.

TP53 mutation analysis. Total RNA was extracted as described previously, and was used as a template for cDNA synthesis (2). The synthesized cDNA was used to perform polymerase chain reaction (PCR) analysis of a high mutation region (aa115-aa342) of the TP53 gene as described previously. The sequence of the PCR products was analyzed by Sanger sequencing (2).

Comprehensive gene expression analysis. Comprehensive gene expression analysis was performed in 5 patients each with and without TP53 mutations (Table I). Biotin-labeled cRNA was synthesized from total RNA according to the Affymetrix manual. Hybridization was performed using a GeneChip Human Genome U133 Plus 2.0 Array (Affymetrix, Santa Clara, CA). A GeneChip Fluidics Station 400 (Affymetrix, Inc., Santa Clara, CA, USA) and Scanner 3000 (Affymetrix, Inc.) were used for detection. Analysis was performed using GeneChip Operating Software (Affymetrix, Inc.) and GeneSpring v7 (Silicon Genetics, Redwood City, CA, USA); the output data were normalized per chip and per gene. Genes with $>3$-fold differential expression between TP53 mutation $(+)$ and (-), that were commonly identified using two parametric tests (Student's t-test and Welch's t-test), were used as gene candidates with differential expression (Fig. 1).

Quantification of mRNA. A quantitative PCR (qPCR) assay was carried out using the SYBR-Green Real-time PCR Master Mix (Life Technologies, Frederick, MD, USA) as described previously (2). The gene expression level was normalized against glyceraldehyde-3-phosphate dehydrogenase (GAPDH) mRNA. Table II lists the primer sequences used.

Immunohistochemistry (IHC). Biopsy tissue was fixed in formalin and embedded in paraffin, and then $4-\mu \mathrm{m}$ thin-sections were prepared. Four cases with p53 wild-type (1T, M11, 7T, M5) and 4 cases with a p53 mutation (M9, M8, M13, 8T) were used. After deparaffinization and removal of endogenous peroxidase, antigen activation was performed using citrate buffer ( $\mathrm{pH} \mathrm{6)}$ ) in a $600 \mathrm{~W}$ microwave oven for 5 min [cystain A (CSTA), stratifin (SFN) or an autoclave for 5 min desmocollin 3 (DSC3)].
Table I. Clinicopathological features and TP53 mutation of 14 cases of maxillary squamous cell carcinoma.

\begin{tabular}{|c|c|c|c|c|c|}
\hline Case & Age & Sex & Stage $^{\mathrm{b}}$ & Grade $^{\mathrm{b}}$ & TP53 mutation ${ }^{c}$ \\
\hline $1 \mathrm{~T}$ & 46 & F & II & 2 & \\
\hline $\mathrm{M}^{\mathrm{a}}$ & 55 & M & III & 1 & \\
\hline $\mathrm{M} 2^{\mathrm{a}}$ & 55 & M & III & 2 & \\
\hline M11 ${ }^{\mathrm{a}}$ & 58 & M & III & 2 & \\
\hline $7 \mathrm{~T}^{\mathrm{a}}$ & 60 & M & III & 2 & \\
\hline M5 ${ }^{\mathrm{a}}$ & 64 & M & III & 3 & \\
\hline M9 ${ }^{\mathrm{a}}$ & 73 & M & III & 1 & P190T \\
\hline M8 & 64 & M & III & 1 & $\mathrm{~F} 285 \mathrm{~K}$ \\
\hline $\mathrm{M} 1^{\mathrm{a}}$ & 63 & M & III & 2 & $\mathrm{R} 280 \mathrm{~S}$ \\
\hline M10 & 64 & M & III & 2 & c. $782+1 \mathrm{G}>\mathrm{A}$ \\
\hline $\mathrm{M} 3^{\mathrm{a}}$ & 51 & M & III & 3 & R156AfsX14 \\
\hline M12 & 80 & M & IV A & 2 & c. $782+1 \mathrm{G}>\mathrm{A}$ \\
\hline M14 & 67 & M & IV A & 2 & Y220C \\
\hline $8 \mathrm{~T}$ & 65 & M & IV A & 2 & H193R \\
\hline
\end{tabular}

${ }^{a}$ Cases used for comprehensive gene analysis. ${ }^{b}$ Based on TMN Classification of Malignant Tumors (7th edition) (14). ${ }^{\mathrm{c}}$ Deletion of wild-type sequence only in case M9.

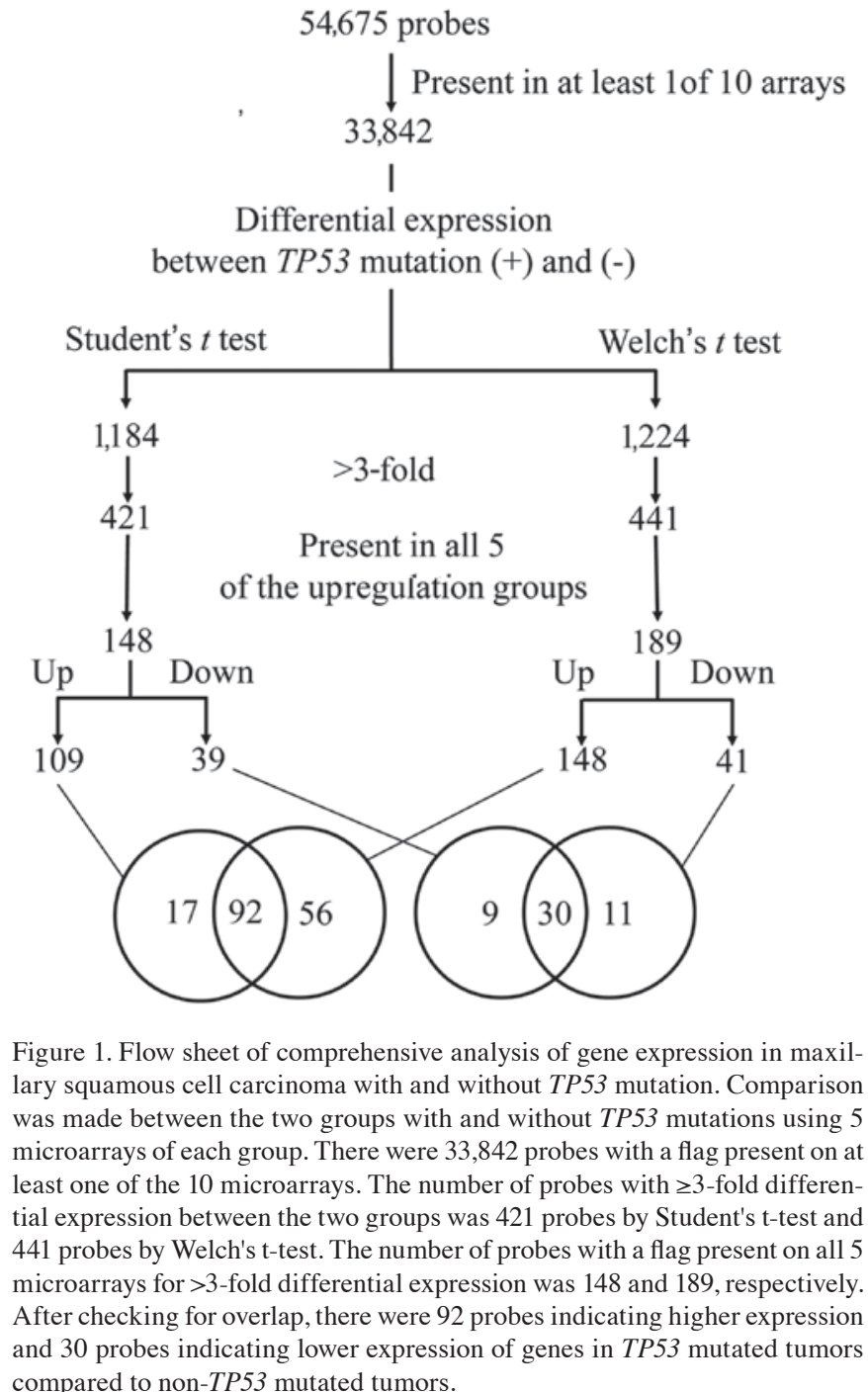
compared to non-TP53 mutated tumors. 
Table II. Primer sequences used for quantitative polymerase chain reaction analysis in this study.

\begin{tabular}{|c|c|c|}
\hline \multirow[b]{2}{*}{ Gene } & \multicolumn{2}{|c|}{ Primer sequence } \\
\hline & Forward (5'-3') & Reverse (5'-3') \\
\hline CSTA & ACGGAAAATTGGAAGCTGTG & TTTGTCCGGGAAGACTTTTG \\
\hline$S F N$ & CAGGCTACTTCTCСССТССТ & TCAATCTCGGTCTTGCACTG \\
\hline DSC3 & ATTGCAGTCTTGATTCTGCC & ACGTTTGTAGGGGAGCACAC \\
\hline GRHL1 & GCTAGTATCAGTCAGATGCA & GAAGGCTCTGATGCGTGATA \\
\hline EPPK1 & TCAGCTCAGCCATAATCACG & ACATGGCCTGGTAGATGCTC \\
\hline PROM2 & CTGATCCCCAGCATCATCTT & ACCAGATCACTCCCACAAGG \\
\hline ANXA8 & AGCTGGTCACAGAGTCTCCT & GCTGCTGAAGGATGTGTGTT \\
\hline CLCA2 & TACCTCTTGCTATTTTGTTA & GCTGCTTTGATGGGAGTAGA \\
\hline SAMD9 & GACATTATGGGCCTGGAAGT & TGTGAATTTCCCCTTTCTGG \\
\hline PRRG4 & AATATTTGTCAGTGCTTAAC & AAATGACCACACAGGCAGAA \\
\hline$D S P$ & TAGGAGAAAATTACCCTCCC & GAAAAGATTGCCGCTGTCAT \\
\hline$F 2 R L 1$ & GCCACTTAGAATAGCATTTG & GATGTGGTCCAAACCCTCTG \\
\hline S100A2 & ATGAGTGGGAATGGCAAGAG & GCAGAGACAGACCCAGGAAG \\
\hline MAST4 & ТСТССТСТCTGTGGGAAGGA & GCCATCTTTGTGGTTCGTTT \\
\hline JUP & AACCAGCTGTCGAAGAAGGA & GTGTCCAGGTCGCTGGTATT \\
\hline$S C D$ & TGTTCGTTGCCACTTTCTTG & TAGTTGTGGAAGCCCTCACC \\
\hline TP63 & GAGGTTGGGCTGTTCATCAT & GAGGAGAATTCGTGGAGCTG \\
\hline KRT6B & TGCGAATGTCCTTTTTAGTT & TAATGGGCAGGATGGTTAGC \\
\hline SFRP4 & GACTTCCGACTTCCTTACAG & TCTGTACCAAAGGGCAAACC \\
\hline HMCN1 & ATCAGCTGAACCACTTATGA & AAACCAAACCTGTCCCACTG \\
\hline MEST & GAATCGATCTGGTCGGCTTA & CATCAGTCGTGTGAGGATGG \\
\hline GAPDH & GGTCGGAGTCAACGGATTTG & GGATCTCGCTCCTGGAAGAT \\
\hline
\end{tabular}

The primary antibodies used were rabbit polyclonal anti-human CSTA IgG $(0.1 \mu \mathrm{g} / \mathrm{ml}$, HPA001031; Atlas Antibodies AB, Stockholm, Sweden), mouse monoclonal anti-human 14-3-3 sigma IgG1 clone 1.N.6 $(1 \mu \mathrm{g} / \mathrm{ml}$, GTX14123; GeneTex, Irvine, CA, USA), mouse monoclonal anti-human desmocollin 3 IgG1 clone Dsc3-U114 $(0.05 \mu \mathrm{g} / \mathrm{ml}$, 61093; Progen Biotech, Heidelberg, Germany), and mouse monoclonal anti-human p53 IgG2b clone DO-7 $(0.69 \mu \mathrm{g} / \mathrm{ml}$, M7001; Dako, Glostrup, Denmark). The reactions were carried out overnight at $4^{\circ} \mathrm{C}$. A Histofine Simple Stain MAX-PO (R) kit or MAX-PO (M) kit (Nichirei, Tokyo, Japan) was used for secondary antibodies. The sections were colored with diaminobenzidine and nuclei were stained with hematoxylin.

Statistical analysis. Age, stage, grade, and mRNA expression levels were compared between the two groups with and without TP53 mutation using the Mann-Whitney $\mathrm{U}$ test. $\mathrm{P}<0.05$ was considered to indicate a statistically significant difference.

\section{Results}

Clinicopathological features of maxillary carcinoma with and without TP53 mutation. Eight of the 14 patients had a TP53 mutation (Table I). These mutations included 5 point mutations, 2 splicing abnormalities, and one frameshift mutation. Table III compares the clinicopathological features of patients with and without TP53 mutations. Tumor stage and grade were not significantly related to TP53 mutation status. However, there
Table III. Comparison of the clinicopathological features of maxillary squamous cell carcinoma with and without TP53 mutation.

\begin{tabular}{lccc}
\hline & \multicolumn{2}{c}{ TP53 mutation } & \\
\cline { 2 - 3 } Feature & $(-)$ & $(+)$ & P-value $^{\text {b }}$ \\
\hline Age $^{\mathrm{a}}$ & $56.3 \pm 6.1$ & $65.9 \pm 8.4$ & 0.0273 \\
Stage & & & 0.0611 \\
II & 1 & 0 & \\
III & 5 & 5 & \\
IVA & 0 & 3 & 0.7047 \\
Grade & & & \\
1 & 1 & 2 & \\
2 & 4 & 4 & \\
3 & 1 & 1 &
\end{tabular}

${ }^{\mathrm{a}}$ Mean \pm standard deviation, ${ }^{\mathrm{b}}$ Two-group comparison using the Mann-Whitney U test.

was a correlation between TP53 mutations and age; thus, TP53 mutation-positive patients were significantly older than those without TP53 mutation ( $\mathrm{P}=0.0273)$. TP53 mRNA expression levels did not significantly differ between the two groups (2). 


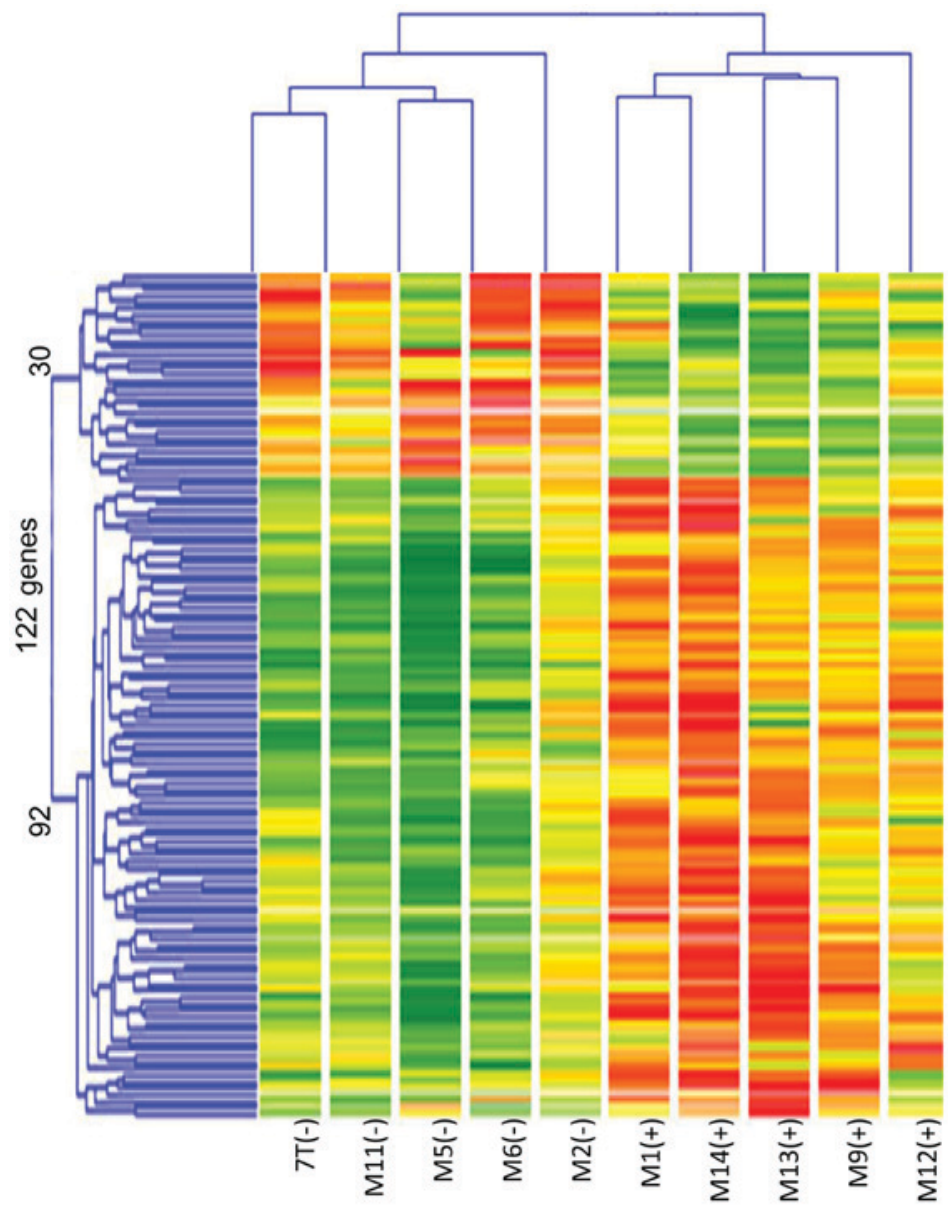

Figure 2. Cluster analysis of 10 maxillary squamous cell carcinomas by their expression profiles of 122 genes. Cluster analysis of 10 maxillary squamous cell carcinomas based on the gene expression pattern of 122 genes with $\geq 3$-fold differential expression in TP53 mutated tumors vs. non-mutated tumors (Gene Spring v7). Minus or plus indicates the absence or presence of TP53 mutation. Red and green colors indicate high and low expression values, respectively.
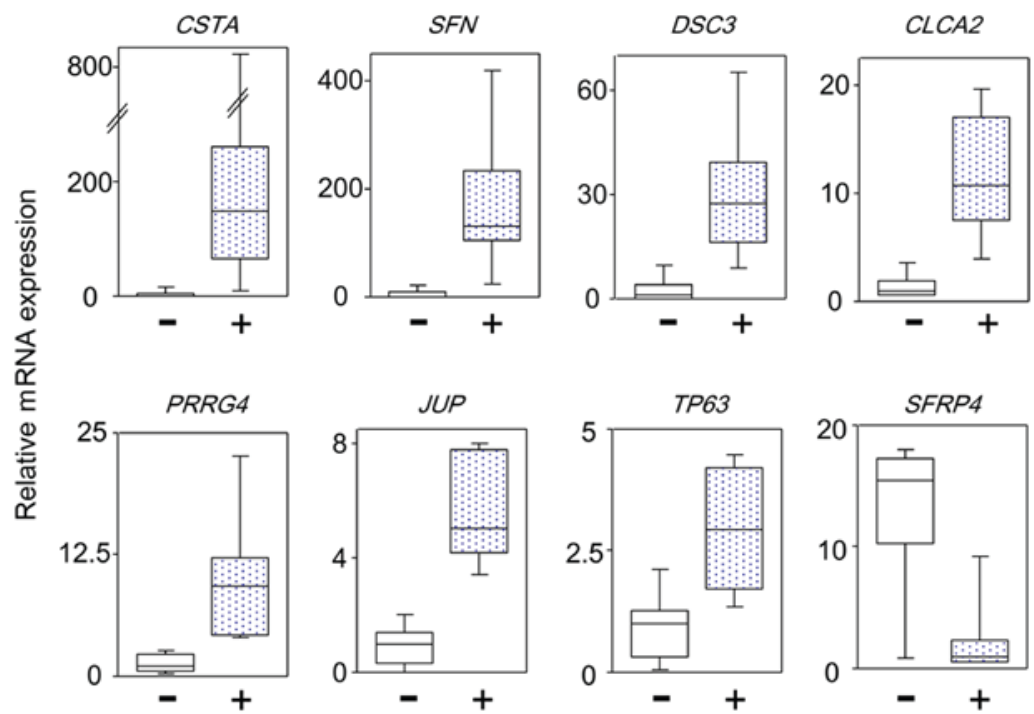

Figure 3. The mRNA expression of 8 representative genes in maxillary squamous cell carcinoma with (+) and without (-) TP53 mutation is shown. Relative mRNA expression is shown, where the mRNA expression of the median level in the low expression group is assigned a value of 1 . The mRNA levels of these 8 representative genes were significantly different between the 2 groups by the Mann-Whitney U test. Each fold-change of difference and the P-value are shown in Table IV.

Differential gene expression in maxillary carcinoma with and without TP53 mutation. Comprehensive gene expression analysis was performed with 10 microarrays using mRNA from the maxillary cancer specimens. The results showed 92 genes in TP53 mutated tumors with $\geq 3$-fold increased expression and 30 genes whose expression was decreased to approximately 
Table IV. Twenty-one validated genes with differential expression in TP53 mutated versus non-mutated tumors.

A, Genes upregulated in TP53 mutated cancer

\begin{tabular}{|c|c|c|c|c|}
\hline Gene symbol & Gene title & $\mathrm{FC}^{\mathrm{a}}$ & P-value & Function \\
\hline CSTA & Cystatin A & 148.8 & 0.0174 & Cysteine protease inhibitor \\
\hline$S F N$ & Stratifin & 129.9 & 0.0106 & $\begin{array}{l}\text { Cell cycle arrest } \\
\text { Tumor progression }\end{array}$ \\
\hline DSC3 & Desmocollin 3 & 27.3 & 0.0106 & Desmosome \\
\hline GRHL1 & Grainyhead-like 1 & 20.1 & 0.0249 & $\begin{array}{l}\text { Transcription factor } \\
\text { Cell adhesion }\end{array}$ \\
\hline EPPK 1 & Epiplakin 1 & 19.9 & 0.0096 & Cell matrix adhesion \\
\hline PROM2 & Prominin 2 & 17.2 & 0.0176 & Membrane glycoprotein \\
\hline ANXA8 & Annexin A8 & 11.7 & 0.0106 & Adherens junction \\
\hline CLCA2 & Chloride channel accessory 2 & 10.7 & 0.0106 & p53-inducible senescence \\
\hline SAMD9 & Sterile alpha motif domain containing 9 & 9.5 & 0.0176 & Regulation of cell proliferation \\
\hline PRRG4 & Proline rich Gla 4 & 9.3 & 0.0062 & Unknown \\
\hline$D S P$ & Desmoplakin & 9.3 & 0.0285 & Desmosome \\
\hline$F 2 R L 1$ & Coagulation factor II receptor-like 1 & 7.5 & 0.0062 & Pro-inflammation \\
\hline S100A2 & S100 calcium binding protein A2 & 7.2 & 0.0176 & Tumor suppressor or promoter \\
\hline MAST4 & $\begin{array}{l}\text { Microtubule associated serine/threonine } \\
\text { kinase family member } 4\end{array}$ & 7.1 & 0.0456 & Unknown \\
\hline$J U P$ & Junction plakoglobin & 5.1 & 0.0007 & Desmosome \\
\hline$S C D$ & Stearoyl-CoA desaturase & 3.2 & 0.0446 & Fatty acid biosynthesis \\
\hline TP63 & Tumor protein p63 & 2.9 & 0.0200 & $\begin{array}{l}\text { ANp63: Cell growth } \\
\text { Tap63: Apoptosis }\end{array}$ \\
\hline KRT6B & Keratin 6B & 2.7 & 0.0285 & Intermediate filament cytoskeleton \\
\hline
\end{tabular}

B, Genes downregulated in TP53 mutated cancer

\begin{tabular}{llrrl}
\hline Gene symbol & \multicolumn{1}{c}{ Gene title } & FC $^{\mathrm{a}}$ & P-value $^{\mathrm{b}}$ & \multicolumn{1}{c}{ Function } \\
\hline SFRP4 & Secreted frizzled-related protein 4 & -15.4 & 0.0112 & Regulation of Wnt signal \\
HMCN1 & Hemicentin 1 & -8.5 & 0.0285 & $\begin{array}{l}\text { Extracellular matrix } \\
\alpha / \beta \text {-fold hydrolase } \\
\text { (imprinting gene) }\end{array}$ \\
\hline
\end{tabular}

${ }^{\text {a}}$ Fold-change (FC). ${ }^{\text {P}} \mathrm{P}$-value, comparison between expression in TP53+ and -mutated cancer groups using the Mann-Whitney U test.

1/3 compared to non-TP53 mutated tumors (Fig. 1). Cluster classification was performed in 10 cases based on the expression pattern of these 122 genes. As shown in Fig. 2, the 10 cases could be accurately classified into two clusters based on TP53 mutation status. To confirm these gene expression levels, qPCR analysis of the mRNA expression of 42 genes with $\geq 4$-fold differential expression between TP53 and non-TP53 mutated tumors based on the microarray results was performed using patient maxillary squamous cell carcinoma samples. Twenty-one genes with significant differences in expression between the two groups were identified. There were no significant differences in the expression level of the remaining 21 genes due to the different normalization methods used. The differential genes commonly identified by different normalization methods are considered to be reliable. Fig. 3 shows representative results of the differential gene expression. Table IV lists 18 genes with high expression and 3 genes with low expression in TP53 mutated tumors compared to non-TP53 mutated tumors. The 18 genes included 8 cell adhesion genes (DSC3; grainyhead like transcription factor 1; epiplakin 1; prominin 2; annexin A8; desmoplakin (DSP); junction plakoglobin $(J U P)$; and keratin 6B) and 4 cell growth inhibition genes ( $S F N$, chloride channel accessory 2, sterile alpha motif domain containing 9, and TP63). Thus, in TP53 mutated tumors, the expression of genes that inhibited proliferation, invasion, and metastases was unexpectedly increased, compared to wild-type tumors.

IHC analysis of DSC3, CSTA, SFN and TP53. IHC analysis of the 3 genes with the highest differential expression between TP53 mutated and non-mutated tumors was performed. Fig. 4 shows representative staining images. CSTA was negative in normal paranasal sinus mucosa, and staining was also weak 


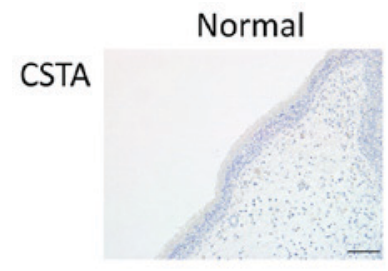

SFN
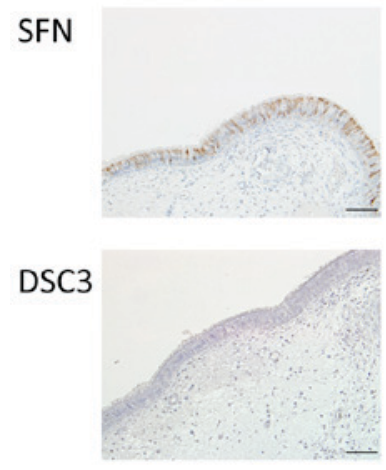

p53

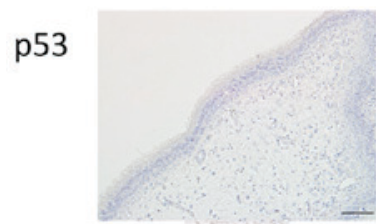

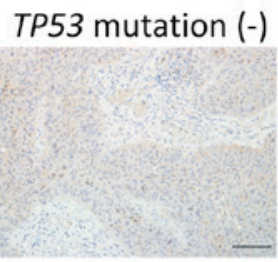
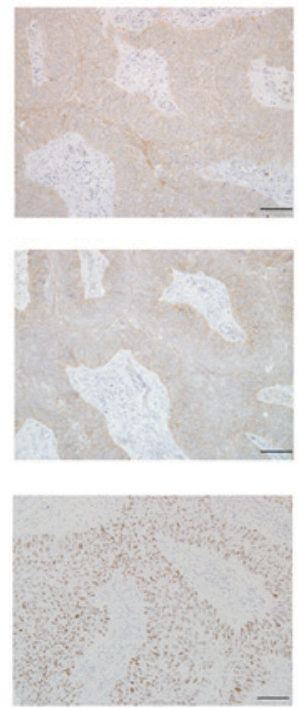

TP53 mutation (+)
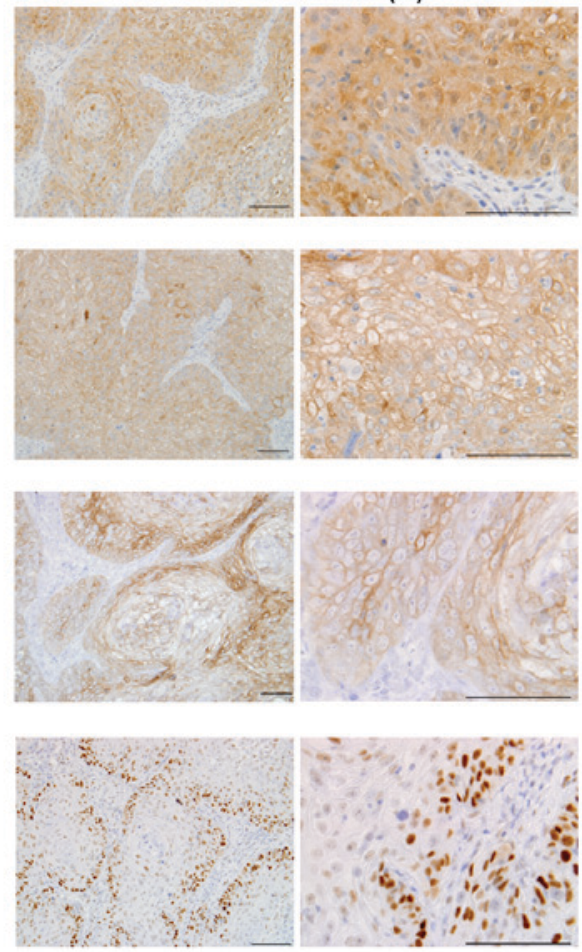

Figure 4. Representative IHC analysis of CSTA, SFN, DSC3, and p53 expression in maxillary squamous cell carcinoma with and without $T p 53$ mutation. Normal, normal paranasal sinus mucosa; TP53 mutation (-), case 1T; TP53 mutation (+), case 8T (CSTA, DSC3) and M9 (SFN). Bar, $100 \mu \mathrm{m}$.

in the TP53 wild-type tumors. In TP53 mutated tumors, CSTA was strongly expressed throughout the entire tumor. SFN was strongly stained in cell membranes in normal paranasal sinus mucosa. Staining of SFN was stronger in mutated tumors than in the wild-type tumors, and was localized more in the cell membrane than in the cytoplasm. DSC3 expression was weakly positive in cell membranes in normal paranasal sinus mucosa. Staining in tumors was stronger in cell membranes, with stronger staining in mutated tumors compared to wild-type tumors. For all these genes, the genes were strongly expressed at the protein as well as at the mRNA level in TP53 mutated tumors.

Localization of the 553 protein was also examined using IHC. p53 expression was negative in normal paranasal sinus mucosa, whereas in TP53 wild-type tumors, positive p53 nuclear expression was observed in all cancer cells. Immunostaining of TP53 mutated tumors did not show p53 staining throughout the tumor; instead, strong nuclear p53 staining was only observed in tumor cells in the margins adjacent to the stroma. The stroma and tumor interior were p53 negative. Even in biopsy specimens after treatment, p53 staining in residual tumor was observed in the tumor margins (data not shown). p53 IHC staining was negative in case M13 (R156AfsX14) because of the frameshift mutation.

\section{Discussion}

This study found clear differences in gene expression between TP53 mutated and TP53 wild-type maxillary squamous cell carcinoma tumors. A characteristic finding was increased expression of cell growth inhibition genes and increased expression of cell adhesion genes such as DSC 3 in the TP53 mutated tumors. Takahashi et al compared gene expression using microarrays in breast cancer with and without TP53 mutations (15). They found that the expression of genes that stimulate the cell cycle and cell division was increased in TP53 mutated tumors, thus suggesting that TP53 mutation was a poor prognostic factor in breast cancer. However, our results were the opposite; i.e., we found increased expression of 8 tumor suppressor genes including SFN in TP53 mutated tumors compared to wild type tumors. In particular, there was $\geq 100$-fold differential expression of CSTA and SFN based on TP53 mutation status (Table IV).

CSTA is a cysteine protease inhibitor that specifically inhibits cathepsin B (16). Cathepsin B, because it localizes on tumor cell surfaces and degrades the extracellular matrix (ECM), is involved in cancer progression (17). Our study showed markedly increased expression of CSTA in TP53 mutated tumors, with strong expression in the cytoplasm. This result suggested that the overexpressed CSTA might more efficiently inhibit ECM degradation, resulting in a decrease in cancer progression of TP53 mutated tumors. Increased CSTA has been shown to inhibit the migration, invasion, and proliferation of laryngeal cancer (18). CSTA expression has been reported to reduce distant metastases in breast cancer, and this may be due to inhibition of cysteine cathepsins (19). On the other hand, increased CSTA was found to be associated with a poorer prognosis in nasopharyngeal cancer (16). Thus, the effects of CSTA differ depending on the type of tumor, and in our study, CSTA may have similar effects as those reported for nasopharyngeal cancer.

SFN is a gene that is induced by TP53 and is also called the 14-3-3 $\sigma$ protein. SFN obstructs G2 cell cycle entry by sequestering $\mathrm{Cdc} 2$-cyclin $\mathrm{B}$ and $\mathrm{Cdc} / \mathrm{Cdk}$ complexes in the 
cytoplasm (20,21). Based on these functions, it was surmised that SFN is a tumor suppressor protein. In addition, 14-3-3 $\sigma$ opposes tumor-promoting metabolic programs by enhancing c-Myc poly-ubiquitination and subsequent degradation. Thus, cancer metabolic reprogramming occurs in tumors with low 14-3-3 o expression (22). However, tumors with high SFN expression have also been reported (23-26). Cancers with high SFN expression have increased proliferation and anti-cancer drug resistance and thus SFN can be regarded as a tumor progressive protein $(23,24)$. Roberts et al reported that SFN binds with plakophilin-3 in desmosomes and decreases incorporation of plakophilin-3 into desmosomes, thereby decreasing desmosomal adhesion and increasing cell migration (27). However, in our study, TP53 mutated tumors were associated with increased expression of DSC3, DSP, and JUP, which are three genes that encode desmosomal structural proteins, suggesting increased cell adhesion. Genes that were overexpressed in the TP53-mutated tumors in our study are often associated with increased adhesion and cell growth inhibition. This means that TP53-mutated tumors may have increased mesenchymal-epithelial transition compared to TP53 wild-type tumors. A difference in the level of mesenchymal-epithelial transition also occurs depending on HPV infection. HPV-positive oropharyngeal squamous cell carcinomas have been reported to lose their epithelial cell phenotype compared with HPV-negative tumors (28). Other studies also have shown that HPV-positive tumors have increased epithelial mesenchymal transition $(29,30)$. HPV-related carcinogenesis is associated with TP53 inactivation, and thus many non-TP53 mutated tumors are HPV positive (13). Therefore, our study findings regarding the expression of genes involved in mesenchymal-epithelial transition in TP53-mutated maxillary carcinoma are consistent with the results of previous studies that showed loss of the epithelial cell phenotype in HPV-positive patients.

TP53 immunostaining in our study showed strong expression in tumor cells regardless of mutation status. Staining was unevenly distributed in TP53 mutated tumors, with negative staining in the tumor center, but strongly positive staining in the tumor margins. This type of uneven distribution has not previously been reported. This staining differed from that of TP53 wild-type tumors in which the entire tumor was uniformly stained. In mutated tumors, TP53 degradation may be more likely, or synthesis may be inhibited, in the tumor center. It is also possible that the interaction of tumor cells with the stroma may affect TP53 expression/localization in mutated tumors. This phenomenon whereby tumor cells with different phenotypes are produced may be linked to chemotherapy resistance. Indeed, regression of mutated tumor also occurs with treatment. However, unlike complete regression of wild-type tumors, mutated tumors have some residual treatment-resistant tumor. This may be due to the heterogeneity of TP53 mutated tumors. Gain-of-function TP53 mutants have recently been shown to have upregulated chromatin regulatory genes that result in genome-wide increases in histone methylation and acetylation. Knockdown or pharmacological inhibition of these chromatin regulatory genes can markedly lower cancer cell proliferation (31).

Mutated TP53 can become a new transcription factor leading to transcription activation that does not occur with wild-type TP53. Our group has shown that this new transcription activity does actually occur. However, the genes with increased expression in the present study mostly played a role in adhesion and cell growth inhibition. Thus this result suggested that TP53 mutation in tumors results in a tumor phenotype that is opposite to that of cancer progression and malignant transformation. As represented by SFN, expression of tumor suppressor genes has in fact been observed in chemotherapy-resistant cancer. The significance of this paradoxical phenomenon will require further investigation.

\section{Acknowledgements}

We thank Miyoko Maeda and Mariko Ishibashi for their assistance with IHC and microarray analysis, respectively.

\section{References}

1. Kelland L: The resurgence of platinum-based cancer chemotherapy. Nat Rev Cancer 7: 573-584, 2007.

2. Kudo I, Esumi M, Kida A and Ikeda M: p53 mutation, but not in vitro predictor genes of therapeutic efficacy of cisplatin, is clinically relevant in comparing partial and complete responder cases of maxillary squamous cell carcinoma. Oncol Rep 24: 851-856, 2010.

3. Bergamaschi D, Gasco M, Hiller L, Sullivan A, Syed N Trigiante G, Yulug I, Merlano M, Numico G, Comino A, et al: p53 polymorphism influences response in cancer chemotherapy via modulation of p73-dependent apoptosis. Cancer Cell 3: 387-402, 2003

4. Liu J, Ma Q, Zhang M, Wang X, Zhang D, Li W, Wang F and Wu E: Alterations of TP53 are associated with a poor outcome for patients with hepatocellular carcinoma: Evidence from a systematic review and meta-analysis. Eur J cancer 48: 2328-2338, 2012.

5. Ohnishi K, Ota I, Takahashi A, Yane K, Matsumoto H and Ohnishi T: Transfection of mutant p53 gene depresses X-ray- or CDDP-induced apoptosis in a human squamous cell carcinoma of the head and neck. Apoptosis 7: 367-372, 2002.

6. Varna M, Bousquet G, Plassa LF, Bertheau P and Janin A: TP53 status and response to treatment in breast cancers. J Biomed Biotechnol 2011: 284584, 2011.

7. Viktorsson K, De Petris L and Lewensohn R: The role of p53 in treatment responses of lung cancer. Biochem Biophys Res Commun 331: 868-880, 2005.

8. Zenz T, Krober A, Scherer K, Habe S, Buhler A, Benner A, Denzel T, Winkler D, Edelmann J, Schwanen C, et al: Monoallelic TP53 inactivation is associated with poor prognosis in chronic lymphocytic leukemia: results from a detailed genetic characterization with long-term follow-up. Blood 112: 3322-3329, 2008.

9. Salani R, Kurman RJ, Giuntoli R II, Gardner G, Bristow R, Wang TL and Shih IM: Assessment of TP53 mutation using purified tissue samples of ovarian serous carcinomas reveals a higher mutation rate than previously reported and does not correlate with drug resistance. Int J Gynecol Cancer 18: 487-491, 2008.

10. Steels E, Paesmans M, Berghmans T, Branle F, Lemaitre F, Mascaux C, Meert AP, Vallot F, Lafitte JJ and Sculier JP: Role of p53 as a prognostic factor for survival in lung cancer: A systematic review of the literature with a meta-analysis. Eur Respir J 18: 705-719, 2001.

11. Stransky N, Egloff AM, Tward AD, Kostic AD, Cibulskis K, Sivachenko A, Kryukov GV, Lawrence MS, Sougnez C, McKenna A, et al: The mutational landscape of head and neck squamous cell carcinoma. Science 333: 1157-1160, 2011.

12. Agrawal N, Frederick MJ, Pickering CR, Bettegowda C, Chang K, Li RJ, Fakhry C, Xie TX, Zhang J, Wang J, et al: Exome sequencing of head and neck squamous cell carcinoma reveals inactivating mutations in NOTCH1. Science 333: 1154-1157, 2011.

13. Westra WH, Taube JM, Poeta ML, Begum S, Sidransky D and Koch WM: Inverse relationship between human papillomavirus-16 infection and disruptive p53 gene mutations in squamous cell carcinoma of the head and neck. Clin Cancer Res 14: 366-369, 2008.

14. International Union against Cancer: TNM classification of malignant tumours. Sobin LH, Gospodarowicz MK and Wittekind C (eds). 7th edition. Wiley-Blackwell, New York, NY, 2009. 
15. Takahashi S, Moriya T, Ishida T, Shibata H, Sasano H, Ohuchi $\mathrm{N}$ and Ishioka C: Prediction of breast cancer prognosis by gene expression profile of TP53 status. Cancer Sci 99: 324-332, 2008.

16. Chang KP, Wu CC, Chen HC, Chen SJ, Peng PH, Tsang NM, Lee LY, Liu SC, Liang Y, Lee YS, et al: Identification of candidate nasopharyngeal carcinoma serum biomarkers by cancer cell secretome and tissue transcriptome analysis: Potential usage of cystatin A for predicting nodal stage and poor prognosis. Proteomics 10: 2644-2660, 2010.

17. Dickinson DP: Cysteine peptidases of mammals: Their biological roles and potential effects in the oral cavity and other tissues in health and disease. Crit Rev Oral Biol Med 13: 238-275, 2002.

18. Li C, Chen L, Wang J, Zhang L, Tang P, Zhai S, Guo W, Yu N, Zhao L, Liu M and Yang S: Expression and clinical significance of cathepsin B and stefin A in laryngeal cancer. Oncol Rep 26: 869-875, 2011.

19. Parker BS, Ciocca DR, Bidwell BN, Gago FE, Fanelli MA, George J, Slavin JL, Moller A, Steel R, Pouliot N, et al: Primary tumour expression of the cysteine cathepsin inhibitor Stefin A inhibits distant metastasis in breast cancer. J Pathol 214: 337-346, 2008.

20. Chan TA, Hermeking H, Lengauer C, Kinzler KW and Vogelstein B: 14-3-3Sigma is required to prevent mitotic catastrophe after DNA damage. Nature 401: 616-620, 1999.

21. Laronga $\mathrm{C}$, Yang HY, Neal $\mathrm{C}$ and Lee MH: Association of the cyclin-dependent kinases and 14-3-3 sigma negatively regulates cell cycle progression. J Biol Chem 275: 23106-23112, 2000.

22. Phan L, Chou PC, Velazquez-Torres G, Samudio I, Parreno K, Huang Y, Tseng C, Vu T, Gully C, Su CH, et al: The cell cycle regulator 14-3-3 $\sigma$ opposes and reverses cancer metabolic reprogramming. Nat Commun 6: 7530, 2015.

23. Perathoner A, Pirkebner D, Brandacher G, Spizzo G, Stadlmann S, Obrist P, Margreiter R and Amberger A: 14-3-3sigma expression is an independent prognostic parameter for poor survival in colorectal carcinoma patients. Clin Cancer Res 11: 3274-3279, 2005 .
24. Neupane D and Korc M: 14-3-3sigma modulates pancreatic cancer cell survival and invasiveness. Clin Cancer Res 14 7614-7623, 2008

25. Nakamura Y, Oshima K, Naoi Y, Nakayama T, Kim SJ, Shimazu K, Shimomura A, Maruyama N, Tamaki Y and Noguchi S: 14-3-3 o expression is associated with poor pathological complete response to neoadjuvant chemotherapy in human breast cancers. Breast Cancer Res Treat 134: 229-236, 2012.

26. Mikami T, Maruyama S, Abé T, Kobayashi T, Yamazaki M, Funayama A, Shingaki S, Kobayashi T, Jun C and Saku T: Keratin 17 is co-expressed with 14-3-3 sigma in oral carcinoma in situ and squamous cell carcinoma and modulates cell proliferation and size but not cell migration. Virchows Arch 466: 559-569, 2015.

27. Roberts BJ, Reddy R and Wahl JK III: Stratifin (14-3-3 o) limits plakophilin-3 exchange with the desmosomal plaque. PloS One 8: e77012, 2013.

28. Hatakeyama H, Mizumachi T, Sakashita T, Kano S, Homma A and Fukuda S: Epithelial-mesenchymal transition in human papillomavirus-positive and -negative oropharyngeal squamous cell carcinoma. Oncol Rep 32: 2673-2679, 2014.

29. Wakisaka N, Yoshida S, Kondo S, Kita M, Sawada-Kitamura S, Endo K, Tsuji A, Nakanish Y, Murono S and Yoshizaki T: Induction of epithelial-mesenchymal transition and loss of podoplanin expression are associated with progression of lymph node metastases in human papillomavirus-related oropharyngeal carcinoma. Histopathology 66: 771-780, 2015.

30. Kranjec C and Banks L: A systematic analysis of human papillomavirus (HPV) E6 PDZ substrates identifies MAGI-1 as a major target of HPV type 16 (HPV-16) and HPV-18 whose loss accompanies disruption of tight junctions. J Virol 85: 1757-1764, 2011.

31. Zhu JJ, Sammons MA, Donahue G, Dou ZX, Vedadi M, Getlik M, Barsyte-Lovejoy D, Al-Awar R, Katona BW, Shilatifard A, et al: Gain-of-function p53 mutants co-opt chromatin pathways to drive cancer growth. Nature 525: 206-211, 2015. 